\title{
Características del síndrome de Guillain-Barré en el área III de salud de la Comunidad Autónoma de Aragón
}

\author{
G. PIÑOL-RIPOLL, P. LARRODÉ PELLICER, M. GARCÉS-REDONDO² I. DE LA \\ PUERTA GONZÁLEZ-MIRÓ ${ }^{1}$, C. IIINIGUEZ MARTÍNEZ
}

Servicios de Neurología y ${ }^{\prime}$ Cardiología. Hospital Clínico. Zaragoza ${ }^{2}$ Servicio de Neurología. Hospital Verge de la Cinta. Tortosa

CHARACTERISTICS OF GUILLAIN-BARRÉ SYNDROME IN THE HEALTHY AREA III OF ARAGON COUNTRY

\begin{abstract}
RESUMEN
Introducción: el síndrome de Guillain-Barré (SGB) es una polineuropatía inflamatoria autoinmune caracterizada por la presencia de parálisis fláccida con arreflexia, trastorno sensorial variable y disociación albúmino-citológica en el LCR.

Pacientes y métodos: estudio retrospectivo de 30 pacientes diagnosticados de SGB entre 1999 y 2005 en el Hospital Clínico de Zaragoza. Se evaluó la incidencia anual, distribución estacional y regional, antecedentes de infección, características clínicas, neurofisiológicas y electrocardiográficas (ECG).

Resultados: la incidencia fue de 1,56/100000 habitantes/año. Mayor frecuencia de eventos en invierno sin alcanzar significación estadística. Mayor frecuencia en sexo masculino $(66,7 \%)$ e incremento de la incidencia con la edad en ambos sexos. La forma de presentación más frecuente fue la paraparesia de extremidades inferiores seguida de la afectación de pares craneales. El $62,5 \%$ recibió tratamiento inmunomodulador. La mortalidad fue del $10 \%$. El $13 \%$ presentó alteraciones autonómicas. Alteraciones electrocardiográficas en el $37 \%$ de los pacientes.

Conclusiones: la incidencia del SGB en Aragón es similar a la que se encontró en otras series. Observamos un aumento de la incidencia con la edad y predilección por los meses de invierno. Elevado porcentaje de pacientes con anomalías ECG mayoritariamente asintomáticas.
\end{abstract}

PALABRAS CLAVE: Aragón. Características clínicas. Incidencia. Inmunoglobulinas. Polineuropatía. Síndrome de Guillain-Barré.

\begin{abstract}
Introduction: Guillain-Barré Syndrome is an acute immune-mediated inflammatory polyneuropathy characterized by flaccid paresia with arreflexia, changeable sensitive disorder and albuminocytologic dissociation in the cerebrospinal fluid (CSF).

Patients and methods: We conducted a retrospective study of $30 \mathrm{GBS}$ patients diagnosed in Hospital Clínico de Zaragoza between 1999 and 2005. Annual incidence, seasonal distribution, preceding acute infection; clinical, electrophysiological and electrocardiographic (ECG) data and evolution were all evaluated.

Results: The incidence was 1.56/100000 inhabitants/year. We observed an upward tendency during winter months. The rates of incidence were higher in men (66.7\%) and increased with age in both sexes. Main clinical presentation form was paraparesia of lower limbs followed on cranial nerve palsy. Immunoglobulin therapy was received by $62.5 \%$ of patients. The rate of death was 10\%. Thirteen per cent of patients had dysautonomia. Electrocardiographic abnormalities were present in $37 \%$ of patients.

Conclusions: GBS incidence in Aragon Country is similar to that found in other studies. An increase with age and an upward tendency during the winter months was observed. High percentage of abnormalities in ECG but the majority of patients was asymptomatic.
\end{abstract}

KEY WORDS: Aragón. Clinical characteristics. Incidence. Immunoglobulin therapy. Polineuropathy. Guillain-Barré Syndrome.

Piñol-Ripoll G, Larrodé Pellicer P, Garcés-Redondo M, de la Puerta González-Miró I, Iñiguez Martínez C. Características del síndrome de Guillain-Barré en el área III de salud de la Comunidad Autónoma de Aragón. An Med Interna (Madrid) 2008; 25: 108-112.

\section{INTRODUCCIÓN}

En la actualidad el SGB se ha convertido en la causa más frecuente de parálisis aguda generalizada (1). Sin embargo fue en el siglo XIX cuando aparecieron los primeros casos en la literatura médica de entumecimiento y debilidad en extremidades y fue en 1859 cuando Jean Baptiste Octave introdujo el término de parálisis aguda ascendente (2). Sin embargo, fueron Charles Guillain y Jean-Alexandre Barré quienes junto con Strohl, publicaron en 1916 un artículo sobre este trastorno donde señalaron la afectación de los reflejos y mencionaron el aumento de proteínas en el líquido cefalorraquídeo (LCR) sin elevación del número de células lo que permitía diferenciar esta enfermedad de otras etiologías frecuentes de la época como la sífilis y la tuberculosis (3). Posteriormente, Miller Fisher concluyó que el caso evaluado por él presentaba aspectos similares o relacionados con el Síndrome de GuillainBarré (SGB) y estableció el concepto de variantes regionales del SGB (4).

Actualmente se puede definir como una polirradiculoneuropatía autoinmune caracterizada clínicamente por la presencia de una parálisis fláccida con arreflexia, trastorno sensorial variable y elevación de las proteínas en el LCR (5). Paralelamente, existen las denominadas variantes regionales como el

Trabajo aceptado: 6 de noviembre de 2007

Correspondencia: Gerard Piñol Ripoll. Servicio de Neurología. Hospital Clínico de Zaragoza. C/ San Juan Bosco, 15. 50009 Zaragoza. e-mail: gerard_437302@hotmail.com 
citado Síndrome de Miller-Fisher que representa aproximadamente un 3\% de los casos (6), parálisis orofaríngea aguda, debilidad faringo-braquio-cervical o la diplejia facial (7).

La incidencia oscila entre 0,6 y 2,4 por cada 100.000 habitantes/año $(8,9)$ con una mortalidad entre el 2 y el $8 \%$ siendo factores de mal pronóstico la edad superior a 40 años, tetraparesia aguda, necesidad de ventilación mecánica y potenciales evocados de baja amplitud (10).

Su etiología es aparentemente autoinmune, desencadenada en ocasiones por una infección vírica o bacteriana ( $C$. jejuni, $H$. influenzae o Citomegalovirus principalmente) que ocasiona autoanticuerpos contra los propios tejidos, lo que se conoce como mimetismo molecular siendo el hallazgo patológico característico la desmielinización inflamatoria multifocal del sistema nervioso periférico que típicamente se inicia en los nódulos de Ranvier donde se concentran los macrófagos encargados de fagocitar la mielina. Sin embargo, todos los hallazgos no pueden explicarse solo por esta hipótesis. Así, el SGB actualmente debe ser considerado como una enfermedad órgano-específica inmunomediada donde estaría implicada tanto la inmunidad humoral como la celular (11).

El propósito de este trabajo es determinar la incidencia y características epidemiológicas, clínicas, inmunológicas, electrofisiológicas y electrocardiográficas del SGB en el área III de salud de Aragón durante el periodo de 1999 al 2005, así como valorar la eficacia y seguridad del tratamiento con inmunoglobulinas.

\section{PACIENTES Y MÉTODOS}

Estudio descriptivo y retrospectivo de los pacientes diagnosticados de SGB y variantes regionales del mismo en el Hospital Clínico de Zaragoza desde el 1 de enero de 1999 hasta 31 de diciembre del 2005. Este es el único hospital responsable del área 3 de salud de Aragón que corresponde a una extensión superior a $6000 \mathrm{~km}^{2}$ compuesto por 116 municipios y un total de 274.533 habitantes, considerando que más del 95\% acuden al servicio de salud público ante una patología potencialmente grave. Por lo tanto, la población de referencia del Hospital Clínico parece representativa del área de estudio.

Todas las historias de pacientes con el diagnóstico de polineuropatía aguda atendidos en los servicios de neurología, medicina interna, neuropediatría y medicina intensiva fueron revisadas retrospectivamente y se seleccionaron aquellas que cumplían los criterios diagnósticos de SGB establecidos por el National Institute of Neurological and Communicative Disorders and Stroke (NINDS) (12).

En todos los pacientes se evaluó la edad, sexo, área de residencia (considerándose áreas rurales aquellas menores de 5000 habitantes), estación del año, factores precedentes (vacunación, proceso infeccioso, cirugía o tóxicos), características clínicas de los enfermos (incluyendo complicaciones sistémicas como insuficiencia respiratoria, SIADH, trastornos autonómicos...), hallazgos electrofisiológicos y electrocardiográficos, evolución y grados de discapacidad en el ingreso y al alta evaluados mediante la escala de Rankin modificada así como los estudios inmunológicos realizados y el tratamiento recibido.

Los estudios de laboratorio incluyeron serologías (virus de Epstein-Barr (VEB), citomegalovirus (CMV), Mycoplasma pneumoniae, virus de la hepatitis B y C, virus herpes zoster
(VHZ) y virus herpes simple (VHS)), estudio de autoanticuerpos (Ac-anti-GQ1B, Ac-anti GM1 y 2) y estudio del líquido cefalorraquideo (LCR) realizado generalmente entre la segunda y cuarta semana. En aquellos casos en que la punción lumbar se realizó durante la primera semana y no aparecieron datos patológicos se repitió en el transcurso de las próximas semanas.

También se han revisado todos los ECG de los pacientes durante el ingreso para poder detectar alteraciones transitorias consecuencia de trastornos autonómicos.

Finalmente, en lo referente a los estudios neurofisiológicos, se realizaron en un tiempo variable que oscila entre la segunda y tercera semana de instauración de la clínica.

Estadísticamente se ha realizado un estudio descriptivo de las distintas variables estudiadas representándose las variables cuantitativas mediante la media aritmética, desviación estándar, máximo y mínimo, mientras que las variables cualitativas se han caracterizado mediante su distribución de frecuencias.

En el análisis de significación se han utilizado los estadísticos de la Chi-cuadrado o el test exacto de Fisher para contrastar diferencias entre variables cualitativas mientras que para comparar con las variables cuantitativas se utilizó el test U Mann-Whitney para datos no paramétricos.

En todos los valores se ha considerado como estadísticamente significativo un valor de $\mathrm{p}<0,05$. Todos los análisis estadísticos han sido realizados con el programa estadístico SPSS (SPSS para Windows, v.13; SPSS, Inc., Chicago, IL).

\section{RESULTADOS}

Durante el periodo de estudio 30 pacientes fueron diagnosticados de SGB.

La edad de los pacientes fue de 50,11 años (SD $=22,18)$, representando los pacientes mayores de 50 años el 52,8\% del total. Por sexos, los varones representaron el $66,7 \%$ de casos con una distribución estacional del $29,6 \%$ en primavera, $18,5 \%$ en verano y otoño, y del $33,4 \%$ en invierno $(p>0,05)$.

La tasa de incidencia cruda fue de 1,56/100.000 habitantes/año. En cuanto a los antecedentes de infección, estuvo presente en el $72 \%$ de los pacientes. De estos, las infecciones respiratorias representaron el grupo más frecuente siendo el $56,25 \%$, seguido de los trastornos gastrointestinales $(37,5 \%)$ y un caso que se atribuyó secundario a infección periodontal. El $31 \%$ de las infecciones respiratorias y entéricas que precedieron al SGB se registraron en invierno. No hemos observado ningún caso secundario a vacunación.

En cuanto a la variabilidad demográfica, y a pesar del importante porcentaje de la población rural de referencia en nuestro hospital, solo el $20,9 \%$ de los pacientes procedían del medio rural.

La mayoría de pacientes $(77,8 \%)$ fueron ingresados en el Servicio de Neurología y el resto en Medicina Interna o Pediatría requiriendo en algunos casos una estancia temporal en la UCI. La estancia media hospitalaria fue de 24,5 días.

De los 30 pacientes tres corresponden a un Síndrome de Miller-Fisher y diplejia facial en dos casos.

En cuanto a las manifestaciones clínicas la forma de presentación más frecuente fue paraparesia en extremidades inferiores $(28 \%)$, afectación de pares craneales $(21 \%)$, tetraparesia $(21 \%)$, paraparesia en extremidades superiores (14\%), 
parestesias (16\%). El 21\% de los pacientes presentaron insuficiencia respiratoria que requirió intubación (todos ellos habían presentado previamente antecedentes de cuadro catarral o gastroenteritis).

En cuanto a las escalas de discapacidad se utilizó las escala de Rankin modificada (Tabla I) en el momento del ingreso y alta con una puntuación de 2,77 (SD = 1,19) y 1,63 (SD = $0,76)$ respectivamente con una mortalidad del $10 \%$.

\section{TABLA I}

\section{ESCALA DE RANKIN MODIFICADA}

-Ningún tipo de síntomas

-Discapacidad no significativa a pesar de los síntomas; capaz de llevar a cabo todas las tareas y actividades habituales

-Discapacidad ligera; incapaz de llevar a cabo todas sus actividades anteriores, pero capaz de cuidar de sus propios asuntos sin ayuda -Discapacidad moderada; requiere alguna asistencia, pero es capaz de andar sin ayuda

-Discapacidad moderadamente severa; incapaz de andar y atender satisfactoriamente sus necesidades corporales sin ayuda

-Discapacidad severa; confinamiento en la cama, incontinencia y requerimiento de cuidados y atenciones constantes

Cuatro pacientes presentaron trastornos autonómicos, en un caso alteraciones vesicales con taquicardia e intensa sudoración. Otro caso asociado a estreñimiento y otros dos casos presentaron dolor torácico de origen coronario que se asociaron a las alteraciones autonómicas. Otros pacientes presentaron cambios electrocardiográficos asintomáticos de modo que en un $42 \%$ (ocho de diecinueve pacientes) de los registros fueron patológicos. Entre los hallazgos encontramos la presencia de alteraciones del ritmo (tres casos de taquicardia y uno de bradicardia sinusal), un paciente con ondas $\mathrm{T}$ picudas sin alteraciones iónicas o metabólicas que lo justificasen, tres enfermos con descenso del ST y 3 pacientes con ondas T negativas patológicas.

Al menos una muestra de LCR fue obtenida en todos los pacientes. El 58\% tenían disociación albúmina-citológica. Entre los pacientes que no la presentaron, en ocho casos se repitió entre la segunda y tercera semana del inicio de los síntomas.

En cuanto a los hallazgos neurofisiológicos, según los criterios de Asbury el $63,1 \%$ de pacientes presentaban una PNP desmielinizante, el $10 \%$ axonal pura y el resto una PNP no clasificable (13).

En cuanto a las complicaciones médicas; dos pacientes fallecieron súbitamente sin poder esclarecerse la causa de la muerte y un paciente falleció por shock séptico secundario a neumonía.

El 62,5\% de los pacientes recibió tratamiento con IG. Ninguno de los pacientes recibió plasmaféresis ante la escasa práctica en nuestro hospital.

En cuanto a las diferencias de la estancia médica se dividieron los pacientes entre aquellos que no habían recibido tratamiento o sólo habían recibido corticoterapia y aquellos que recibieron tratamiento con $\mathrm{Ig} \mathrm{IV}$, no encontrando diferencias estadísticamente significativas en cuanto a estancia media hospitalaria $(\mathrm{p}=0.622)$, encontrando mayor discapacidad al ingreso en aquellos pacientes que recibieron tratamiento con inmunoglobulinas. Ninguno de los pacientes tratados con Ig IV sufrió efectos secundarios.

\section{DISCUSIÓN}

El SGB es la causa más frecuente de parálisis fláccida aguda, pudiendo presentarse a cualquier edad y en ambos sexos. En nuestra serie no encontramos diferencias en cuanto a la preferencia estacional, mostrándose un discreto aumento en la frecuencia durante los meses de invierno, de acuerdo con lo publicado en la literatura (14). En cuanto a la distribución por edades, encontramos una mayor incidencia en mayores de 50 años, confirmando datos que hablan de que la presentación de éste síndrome es más raro en la lactancia mientras que hay un ligero aumento del riesgo en la adolescencia y en la tercera edad (15). De acuerdo con otros estudios, observamos una predilección por el sexo masculino (16).

El $72 \%$ de los pacientes presentó antecedentes de procesos infecciosos, destacando que el 37,5\% correspondieron a alteraciones gastrointestinales con cifras similares al artículo de Aladro-Benito y cols. (10) pero superior a lo mostrado en otras series. En todos los pacientes, excepto en dos, se realizó estudio de serologías encontrando sólo un caso compatible con infección aguda por VEB. A pesar de realizar coprocultivos en todos los pacientes con síntomas gastrointestinales en ninguno de ellos se obtuvieron resultados positivos.

En cuanto a los hallazgos clínicos, LCR y electrofisiológicos son similares a los reportados en otras series.

La mayoría de los pacientes, en algún momento de la enfermedad desarrollan disociación albúmina-citológica, pero la concentración de proteínas se mantiene normal en algunos casos a lo largo de toda la evolución del proceso, por lo cual, actualmente, la mayor indicación de la punción lumbar sería la exclusión de otras entidades como el SGB en el curso de una infección por VIH (17).

A pesar que los estudios de conducción son poco sensibles en los primeros días de la enfermedad, de modo que los parámetros más alterados durante estos primeros días son la respuesta $\mathrm{F}$ y el reflejo $\mathrm{H}$, así como la disminución de la amplitud del potencial motor (18) y que incluso en la mitad de los casos no muestran alteraciones en este estadio; los estudios electrofisiológicos constituyen el test diagnóstico de elección (1) por detrás de la sospecha clínica.

Precisamente desde el punto de vista clínico, el déficit motor suele ser asimétrico, usualmente comienza por las extremidades inferiores y se extiende con posterioridad hacia los brazos y las estructuras inervadas por los nervios craneales en un plazo de una a tres semanas (19).

Lo que marca la debilidad clínica definitiva es el grado de lesión axonal y no la intensidad de la desmielinización. Por ello, las formas con degeneración axonal tienen peor pronóstico que la forma clásica desmielinizante; tardan más en recuperarse de la debilidad y pueden hacerlo de forma incompleta, mientras que los parámetros de velocidad de conducción en cualquiera de los tramos nerviosos no tienen significado pronóstico.

A pesar de la baja frecuencia de aparición de síntomas autonómicos sintomáticos en nuestra serie encontramos una elevada frecuencia de alteraciones electrocardiográficas asintomáticas posiblemente secundaria a disfunción autonómica. 
Los trastornos disautonómicos aunque pueden presentarse en pacientes con afectación ligera suelen ser más frecuentes en aquellos casos donde el déficit motor es más grave, en los enfermos con fallo respiratorio y en las formas axonales. Las alteraciones más frecuentes son las arritmias cardíacas, íleo paralítico, disfunción vesical, parálisis de la acomodación y cambios en la tensión arterial y electrocardiográficos (20). Dentro de éstas alteraciones autonómicas, un aspecto importante a destacar de nuestra serie y poco valorado en los diversos estudios son las alteraciones electrocardiográficas que presentan estos pacientes, los mecanismos subyacentes a las cuales no han sido completamente explicados (21). Los trastornos del ritmo (la taquicardia sinusal persistente es la más frecuente), siendo la disminución del intervalo R-R y la presencia de HTA grave los principales factores predictivos para el desarrollo de arritmias (22). Sin embargo, las bradiarritmias suelen considerarse como los trastornos cardiovasculares más peligrosos y pueden incluir la asistolia, siendo trastornos que pueden desencadenarse por la presencia o ausencia de estímulos vagotónicos pudiendo requerir el empleo de marcapasos temporal o permanente.

En una serie de 16 pacientes ingresados en UCI las alteraciones del ritmo cardíaco fueron observadas en 13 de ellos entre las que se incluían bradiarritmia, bloqueos sinusales y auriculoventriculares y/o taquicardiacas supraventriculares o ventriculares (23). En nuestra muestra encontramos hallazgos patológicos en el $42 \%$ de pacientes teniendo en cuenta que no se trata solo de enfermos con extrema afectación como en el estudio de Greenland y cols. sino que recoge enfermos en los distintos espectros de gravedad de la enfermedad. A pesar de que en las distintas series se considera el patrón de afectación axonal como responsable principal de aparición de patología cardiaca sólo uno de nuestros pacientes con afectación electrocardiográfica presentaba este patrón.

En un intento de identificar aquellos pacientes con más alto riesgo de desarrollar alteraciones del ritmo algunos autores proponen la realización de medidas seriadas del intervalo $\mathrm{R}-\mathrm{R}$ en pacientes con enfermedad severa afectando el tronco y la musculatura respiratoria (24).
A pesar de todas estas precauciones, todos aquellos enfermos que presenten asociadas alteraciones electrocardiográficas deben requerir el diagnóstico diferencial con la Enfermedad de los legionarios o porfiria aguda intermitente en caso de asociarse clínica de infección respiratoria en el primer caso o dolor abdominal, nauseas o vómitos en el segundo caso.

En cuanto al tratamiento, debido a la escasa experiencia en nuestro centro con la plasmaféresis se instauró tratamiento con Ig IV. Dos estudios han demostrado que su uso es tan efectivo como la plasmaféresis y, en comparación con la misma o empleando la Ig IV consecutivamente a ésta no existen diferencias significativas $(25,26)$. La dosis utilizada fue la recomendada en los distintos estudios que corresponde a una dosis de $0,4 \mathrm{~g} / \mathrm{kg} /$ día durante 5 días.

La administración de IG IV constituye una modalidad terapéutica generalmente bien tolerada. Entre los efectos adversos más frecuentes encontramos: reacciones pirógenas, fiebre, escalofríos, cefalea, mialgias, taquicardia, nauseas y disnea; mientras que en pacientes con déficit de Ig A puede ocasionar una reacción de hipersensibilidad incluyendo anafilaxia e incluso precipitar fallo cardíaco en pacientes con insuficiencia cardíaca congestiva (27). En nuestra serie destacamos la seguridad del fármaco ante la ausencia de efectos secundarios.

El tratamiento esteroideo se abolió tras demostrarse su ineficacia. Sin embargo, la asociación de metilprednisolona con Ig IV podría tener un efecto beneficioso a corto plazo sin modificar el pronóstico a largo plazo $(28,29)$.

A pesar de que nuestro trabajo presenta las limitaciones propias de los estudios retrospectivos la recogida selectiva de los pacientes y los datos de incidencia similares a las encontradas en otros estudios retrospectivos y prospectivos incrementan el valor de nuestro estudio (30).

De modo que podemos concluir que la incidencia anual cruda de nuestra comunidad es del 1,56/100000 habitantes, con una elevada frecuencia de antecedentes infecciosos y con mayor afectación del sexo masculino. Destacamos la importancia en la detección de alteraciones cardiológicas para intentar reducir la mortalidad que se encuentra alrededor del $10 \%$ en nuestra serie.

\section{Bibliografía}

1. Ropper AH. The Guillain-Barré syndrome. N Engl J Med 1992; 326: 1130-6.

2. Landry O. Note sur la paralysie ascendante aigue. Gazette Hebdomadaire Méd Chir 1859; 6: 272-88

3. Guillain G, Barré JA, Strohl A. Sur un syndrome de radiculo-névrite avec hyperalbuminose du liquide céphalo-rachidien sans reaction cellulaire remarques sur les caracterès cliniques et graphiques des réflexes tendineux. Bull Soc Méd Hop Paris 1916; 40: 1462-70.

4. Fisher CM. An unusual variant of acute idiopathic polyneuritis (syndrome of ophtalmoplegia, ataxia and areflexia). N engl J Med 1956; 40: 1462-70.

5. Asbury AK. New concepts of Guillain-Barré syndrome. J Child Neurol 2000; 15: 183-91.

6. Ropper AH, Wijdicks EFM, Truax BT. Guillain-Barré Syndrome. Philadelphia, Pa: FA Davis Co Publishers; 1992.

7. González-Santiago MP, Cebrero M, Lassaletta A, García-Frías E. Parálisis facial bilateral como forma de presentación de síndrome de Guillain-Barré. An Pediatr 2003; 58 (1): 77-88.

8. Rees JH, Thompson RD, Smeeton NC, Hugues RAC. Epidemiological study of Guillain-Barré syndrome in south east England. J Neurol Neurosurg Psychiatry 1998: 64; 74-7.

9. Matías-Guiu J, Martín R, Blanquer J, González MJ, Falip R, Oltra A Incidence of Guillain-Barré síndrome in Spain, 1985-1997: Epidemiological and public health views. Eur Neurol 2001; 46: 83-91.

10. Aladro-Benito Y, Conde-Sendín MA, Muñoz-Fernández C, Pérez-Correa S, Alemany-Rodríguez MJ, Fiuza-Pérez MD, Álamo-Santana F. Síndrome de Guillain-Barré en el área norte de Gran Canaria e isla de Lanzarote. Rev Neurol 2002; 35 (8): 705-710.

11. Kieseier BC, Keifer R, Gold R, Hemmer B, Willison H, Hartung HP. Advances in understanding and treatment of immune-mediated disorders of the peripheral nervous sytem. Muscle Nerve 2004; 30: 131-56.

12. National Institute of Neurologic and Communicative Disorders and Stroke: Criteria for diagnosis of Guillain-Barré syndrome. Ann Neurol 1978; 3: 565-6

13. Asbury AK, Cornblath DR. Assessment of current diagnostic criteria for Guillain-Barré syndrome. Ann Neurol 1990; 27 (Supl.): S21-4. 
14. Pascual-Pascual SI. Aspectos actuales de las neuropatías inflamatorias agudas y crónicas. Síndrome de Guillain-Barré y polineuritis crónica inflamatoria desmielinizante. Rev Neurol 2002; 35 (3): 269-276.

15. Hahn AF. Guillain-Barré síndrome. Lancet 1998;352:635-41.

16. Cuadrado JI, de Pedro-Cuesta J, Ara JR, Cemillan CA, Díaz M, Duarte J, et al. Guillain-Barré syndrome in Spain. Eur Neurol 2001; 46: 83-91.

17. Cornblath DR, McArthur JC, Kennedy PG, Witte AS, Griffin JW. Inflamatory demyelinating peripheral neuropathies associated with human Tcell lymphotropic virus type III infection. Ann Neurol 1987; 21: 32-40.

18. Gordon PH, Wilborn AJ. Early electrodiagnostic findings in GuillainBarré síndrome. Arch Neurol 2001; 58: 913-7.

19. McKhann GM. Guillain-Barré Syndrome: Clinical and therapeutic observations. Ann Neurol 1990; 27 (Supl.): S13-6.

20. Dalos NP, Borel C, Hanley DF. Cardiovascular autonomic dysfunction in Guillain-Barré syndrome. Therapeutic implications of Swan-Ganz monitoring. Arch Neurol 1988; 45: 115-7.

21. Palferman TG, Wright I, Doyle DV. Electrocardiographic abnormalities and autonomic dysfunction in Guillain-Barré syndrome. Br. Med. J. 1982; 284: 1231-23.

22. Winer JB, Hugues RA. Identification of patients at risk of arrhythmia in the Guillain-Barré syndrome. Q J Med 1998; 68: 735-9.

23. Greenland P, Griggs R. Arrhytmic complications in the Guillain-Barré
Syndrome. Arch Intern Med 1980;140: 1053-5.

24. Oakley CM. The Heart in the Guillain-Barré syndrome. Br. Med. J. 1982; 288: 94.

25. Van der Meche FG, Schmitz PI. A randomized trial comparing intravenous immune globulin and plasma Exchange in Guillain-Barré syndrome. Dutch Guillain-Barré Study Group. N Engl J Med 1992; 326: 1123 9. Plasma Exchange/sandoglobulin Guillain-Barré Síndrome Trial Group.

26. Randomised trial of plasma exchange, intravenuos immunoglobulin, and combined treatments in Guillain-Barré syndrome. Lancet 1997; 349: 225-30.

27. Van der Mece FG, Van Doorn PA. Guillain-Barré Síndrome. Curr Treta Options Neurol 2000; 2: 507-16.

28. The Dutch Guillain-Barré Study Group. Treatment of Guillain-Barré síndrome with high-dose immune globulins combined with methylprednisolone: a pilot study. Ann Neurol 1994; 35: 749-52.

29. Hugues RA, Swan AV, van Koningveld R, van Doorn PA. Corticosteroids for Guillain-Barré Syndrome. Cochrane Database Syst Rev 2006; 19 (2): CD001446.

30. Rees JH, Thompson RD, Smeeton NC, Hugues RAC. Epidemiological study of Guillain-Barré syndrome in south east England. J Neurol Neurosurg Psychiatry 1998; 64: 74-7. 